\title{
Collapse of Growth Cone Structure on Contact with Specific Neurites in Culture
}

\author{
Josef P. Kapfhammer and Jonathan A. Raper \\ Max-Planck-Institut für Entwicklungsbiologie, 7400 Tübingen, West Germany
}

\begin{abstract}
We have studied the morphology of embryonic chick retinal and sympathetic growth cones as they meet retinal and sympathetic neurites grown in culture. Growth cones preserve their normal morphology and ability to locomote when retinal growth cones contact retinal neurites or when sympathetic growth cones contact sympathetic neurites. Growth cones collapse and their motility ceases when retinal growth cones contact sympathetic neurites or when sympathetic growth cones contact retinal neurites. Collapse was never observed before a growth cone touched a neurite. As a growth cone collapses, the neurite it leads retracts. After a brief pause, a new growth cone is organized and extension recommences. These results suggest that contact-mediated inhibition of locomotion could play a role in growth cone guidance.
\end{abstract}

We have examined the behavior of embryonic chick retinal and sympathetic growth cones as they collide with retinal and sympathetic neurites in culture (Kapfhammer et al., 1986). We used a quantitative analysis of encounters between individual growth cones and neurites to show that retinal growth cones cross retinal neurites more easily than they cross sympathetic neurites, while sympathetic growth cones cross sympathetic neurites more easily than they cross retinal neurites. These results imply that there is at least one specific difference between retinal and sympathetic neurites that allows growth cones to distinguish between them. They also imply that retinal and sympathetic growth cones are programmed to react differently upon confronting the same neurites.

In this paper we examine these growth cone-neurite encounters with time-lapse videos taken at high magnification and with high time resolution. This has allowed us to examine the detailed morphology of retinal and sympathetic growth cones as they interact with retinal and sympathetic neurites.

We have found that when retinal growth cones contact retinal neurites or sympathetic growth cones contact sympathetic neurites, growth cones maintain their normal, flattened, motile morphology. However, when retinal growth cones contact sympathetic neurites or sympathetic growth cones contact retinal neurites, growth cones lose their flattened, motile morphology.

\footnotetext{
Received Jan. 23, 1986; revised May 22, 1986; accepted June 12, 1986.

We thank Susannah Chang Simon Goodman and Rupert Hallmann for their criticism of this manuscript. We thank Friedrich Bonhoeffer for sharing his knowledge and video equipment with us. We also thank Barbara Grunewald for technical assistance. This work was supported by the Max-Planck-Gesellschaft. J.P.K. received a stipend from the Studienstiftung des deutschen Volkes.

Correspondence should be addressed to Jonathan A. Raper, MPI Entwicklungsbiologie, Spemannstrasse 35, 7400 Tübingen, West Germany.

Copyright (C) 1987 Society for Neuroscience $0270-6474 / 87 / 010201-12 \$ 02.00 / 0$
}

As their structure collapses, the neurites they lead retract. These results imply that the motility of particular growth cones is shut down by contact with specific neurites.

\section{Materials and Methods}

Cultures. Chick retinal (embryonic day 6) and sympathetic (embryonic day 8) explants were cultured on a laminin-coated glass substrate according to the methods described in Kapfhammer et al. (1986). Heartconditioned medium (HCM) was sometimes used to improve sympathetic growth. HCM (Nishi and Berg, 1977) was made by growing dissociated embryonic day 8 heart cells $\left(5 \times 10^{5} / \mathrm{ml}\right)$ in F12 medium on tissue-culture plastic (Greiner). This medium was supplemented with $5 \%$ fetal calf serum (Gibco), 5\% chicken serum (Gibco), glucose, glutamine, penicillin, and streptomycin. The medium was harvested after $24 \mathrm{hr}$ in culture, filtered, and further supplemented so that the final concentrations of all components were the same as described in Kapfhammer et al. (1986). Plastic culture dishes were modified so we could film directly through the coverslips on which the cultured explants were growing. Holes were cut in the bottoms of the dishes, and laminincoated coverslips were cemented over the holes with silicon grease. After approximately $1 \mathrm{~d}$ in culture, individual culture dishes were transferred onto the stage of an inverted Zeiss Axiomat microscope. The entire microscope was enclosed in a plastic curtain and maintained at $37^{\circ} \mathrm{C}$ by a heater. Humidified $5 \% \mathrm{CO}_{2}$ was delivered to the cultures as described in Kapfhammer et al. (1986).

Optics and video time lapse. Growth cones were viewed in phase contrast through a $50 \times$ Planapo lens (Zeiss). The image was further magnified $2-3.2 \times$ by the Axiomat's Optovar system. This yielded a field of vision on the video screen of approximately $90 \times 90 \mu \mathrm{m}$ at the 2 -fold magnification generally used. Individual filopodia were resolved clearly. A time-lapse video recording system was fitted to the microscope. It consisted of a Hamamatsu C 1000 video camera, a camera control unit with adjustable beam and gain controls, a Siemens Sirecord S time-lapse video recorder, a National WJ-810 time-date generator, and two Grundig BG 330 video monitors. The microscope was also equipped with a $35 \mathrm{~mm}$ camera for microphotography.

Data collection. All video recordings were made of cultures that were 24-36 hr old, when the neurites from the 2 confronted explants were just beginning to meet. The identities of individual growth cones and neurites were determined before filming (and before they met) by tracing them back to their explants of origin. Situations were chosen in which individual growth cones approached single neurites or a small fascicle of neurites at an angle close to $90^{\circ}$. A video image was recorded every second. This yielded an acceleration factor of $50 \times$, which gives a time resolution good enough for resolving even fast filopodial and lamellipodial movements. In addition, $35 \mathrm{~mm}$ photographs were taken at selected times.

Figure 9 is constructed from tracings of photographs taken from the video screen. Photographs from the screen were made at selected intervals and printed at magnifications that compensated for the differing magnifications of the video images. The positions and rough shapes of neurites and growth cones were traced onto transparent paper.

\section{Results}

Growth cone morphology

Normal, translocating retinal and sympathetic growth cones have similar but distinguishable morphologies. Retinal growth cones 
Figure 1. Phase micrographs of a retinal growth cone $(A)$ and a sympathetic growth cone $(B)$ cultured on laminin. Calibration bar, $10 \mu \mathrm{m}$.

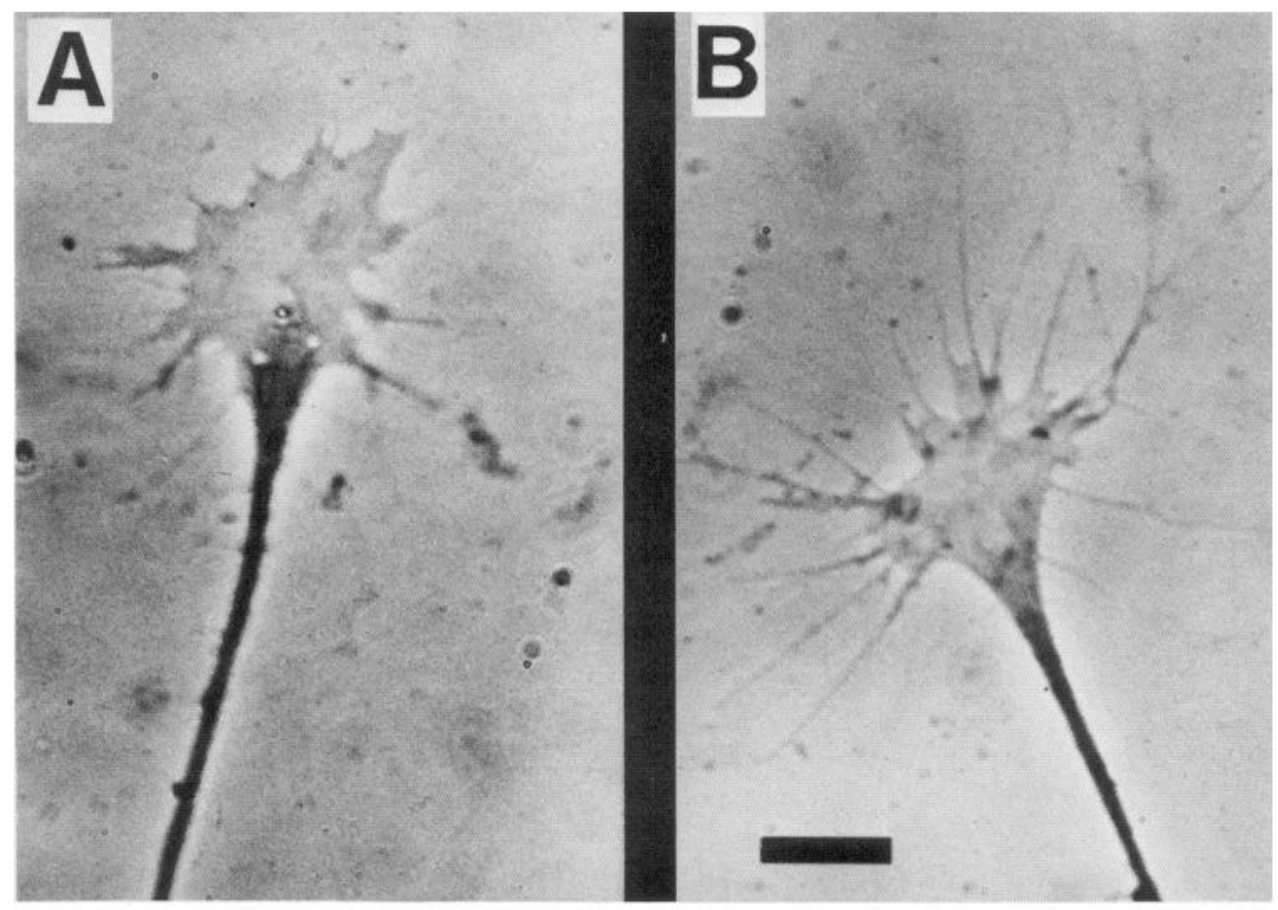

(Fig. 1 $A$ ) generally have 1 large flattened region, at times as large as $25 \mu \mathrm{m}$ across. The leading edge of this flattened region generally has many short, stubby projections radiating away from its center. Long thin filopodia are rare (F. Bonhoeffer, personal communication). These observations indicate that growth cones are able to translocate efficiently without filopodia. Sympathetic growth cones in the same culture conditions also have a large flattened region that can be as much as $25 \mu \mathrm{m}$ in diameter (Fig. $1 B$ ). Many long, fine filopodia project from it, covering an area of roughly $1000 \mu \mathrm{m}^{2}$.

\section{Retinal growth cones meeting retinal neurites}

An encounter between a retinal growth cone and a small fascicle of retinal neurites is shown in Figure 2. The retinal growth cone approaches the fascicle rapidly (Fig. 2, $A, B$ ), touches it, and immediately makes extensive contact (Fig. $2 C$ ). In Figure $2 D$, crossing is nearly complete, although a broad area of adhesion remains between the growth cone and neurite fascicle (open arrowhead). Five minutes later, the growth cone has crossed the fascicle and is extending rapidly away from it (Fig. 2, $E, F$ ). Throughout the entire interaction, the growth cone advances at a steady pace and maintains its normal flattened morphology. The growth cone generally ignores the retinal fascicle except to briefly adhere to it. It should be noted that retinal growth cones can extend on retinal neurites. This is demonstrated by the slow advance of a retinal growth cone on the fascicle (closed arrowheads, Fig. 2, $B-E$ ).

\section{Sympathetic growth cones meeting sympathetic neurites}

An encounter between a sympathetic growth cone and a sympathetic neurite is shown in Figure 3. As is generally the case, this sympathetic growth cone is extending more slowly than most retinal growth cones (Fig. 3, $A, B$ ). Filopodial contact between the growth cone and the neurite is established early, but note that the neurite is not much deformed (Fig. $3 B$ ). Within 10 min massive contact is established between the growth cone and the neurite, and the leading edge of the growth cone has already crossed the neurite (Fig. $3 C$ ). A third sympathetic neurite has extended along the original sympathetic neurite from the left. This neurite is pulled towards the sympathetic growth cone (arrowhead). The growth cone continues to advance, pulling the original neurite downwards (Fig. 3, $C-E$ ), then upwards (Fig. 3, $F-H$; arrowheads). Seventy-five minutes after the first contact, the growth cone has completely crossed the neurite (Fig. $3 I$ ). Only a few thin processes originating from the shaft behind the growth cone are still in contact with the neurite (arrowheads in Fig. 3I).

The sympathetic growth cone continues to advance, maintaining its normal flattened morphology throughout the entire encounter. In contrast to the retina-retina encounter shown in Figure 2, the adherence of the sympathetic growth cone to the sympathetic neurite is tight enough and exerts sufficient tension to deform it. This suggests that the difference between sympathetic-sympathetic adhesion and sympathetic-laminin adhesion is greater than that between retinal-retinal adhesion and retinal-laminin adhesion. This finding is in agreement with the observation that sympathetic neurites are more fasciculated than are retinal neurites grown on the same laminin substrate (see Fig. 1 in Kapfhammer et al., 1986).

\section{Retinal growth cones meeting sympathetic neurites}

An encounter between a retinal growth cone and a sympathetic neurite is shown in Figure 4. Before contact (Fig. 4A), the retinal growth cone approaches the neurite rapidly. The flattened region of the growth cone maintains its normal motile morphology even as it first contacts the neurite (Fig. $4 B$ ). Three minutes later, the growth cone's morphology is altered (Fig. 4C). The flattened region is thicker and reduced in size. The filopodia, especially near the base of the growth cone, are broader and shorter. Over the next $9 \mathrm{~min}$, the structure of the entire growth cone collapses and is resorbed into the retinal neurite behind it (Fig. 4D). A few strands of stretched tissue (arrowhead) bridge 


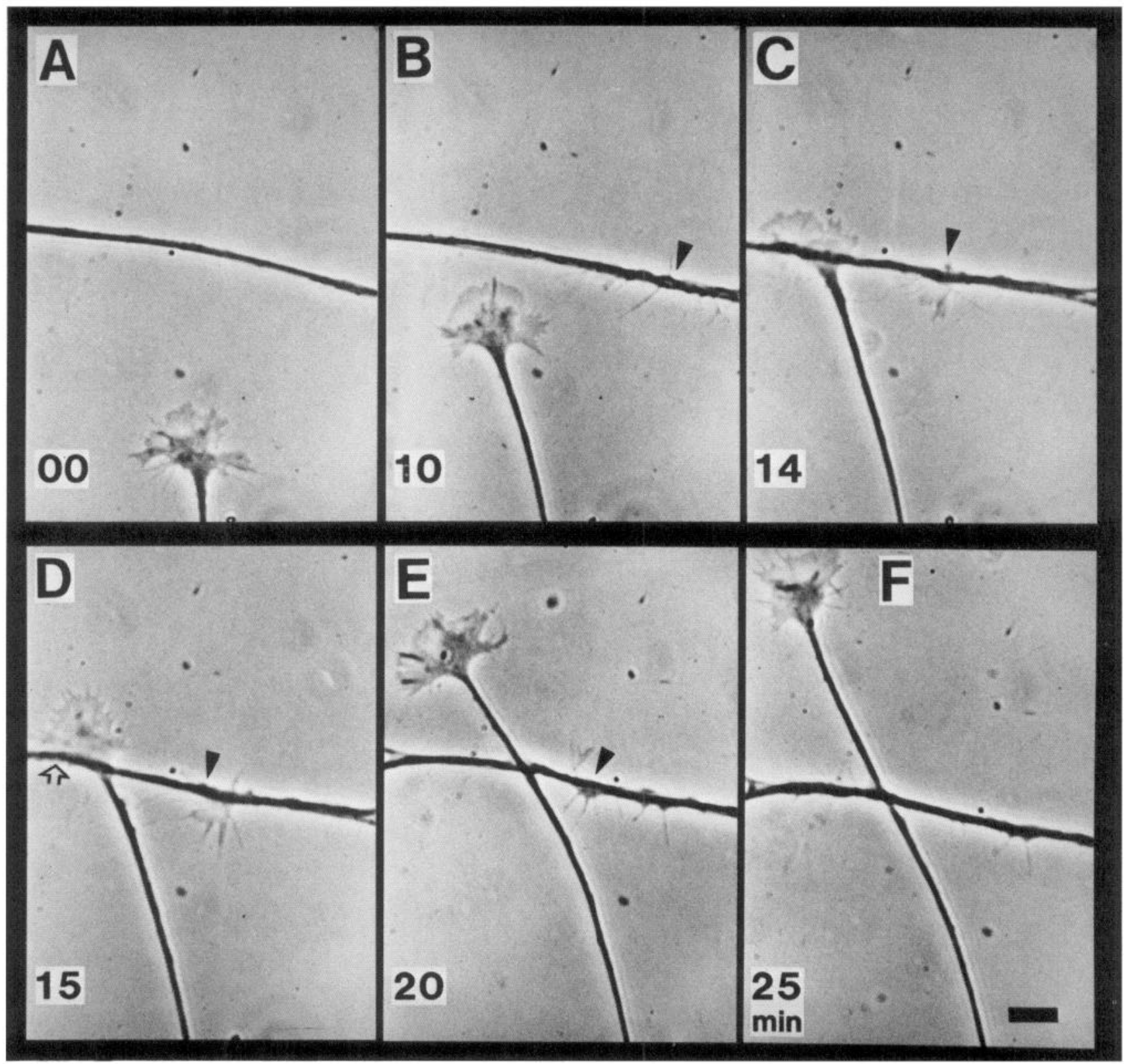

Figure 2. Retinal growth cone crossing a small fascicle of retinal neurites. Field shifts to the left between $D$ and $E$. Time in minutes indicated at lower left of each frame. Calibration bar, $10 \mu \mathrm{m}$.

the gap between the sympathetic neurite and the retinal neurite as the retinal neurite retracts backwards. Note that the sympathetic neurite is displaced towards the retinal neurite during the retraction (stars indicate an arbitrary fixed point). One cause for retinal retraction may be that tension accumulates in the neurite as a healthy growth cone advances and this tension pulls the collapsing growth cone backwards as it loses its attachment to the substrate. During this phase of the retraction cycle, the retinal neurite appears to be held in place, at least in part, by the retinal strands spanning the gap between the retinal and sympathetic neurites.

Retinal retraction is greatest $11 \mathrm{~min}$ later (Fig. $4 E$ ); however, motile activity is recommencing behind the neurite's tip (arrowhead). Five minutes later, a new growth cone has been organized and is reapproaching the sympathetic neurite (Fig. $4 F$ ).
This second advance occurs even in the presence of the cytoplasmic bridges remaining from the first contact and retraction. A process still connects the new growth cone to the initial contact site on the sympathetic neurite. The newly formed growth cone contacts the sympathetic neurite (Fig. $4 G$ ) and soon afterwards shows the same initial signs of collapse as were seen during the first contact (compare Fig. 4, $C$ and $H$ ). The retinal growth cone once again collapses and retracts. Yet another growth cone is organized and begins to extend in a new direction (Fig. 4I). Two retinal processes remain firmly attached to the sites where the previous retinal growth cones contacted the sympathetic neurite.

Another example of a retinal growth cone encountering a sympathetic neurite is shown in Figure 5. As in the previous example, the retinal growth cone advances rapidly and makes extensive contact with the sympathetic neurite (Fig. 5, $A, B$ ). 


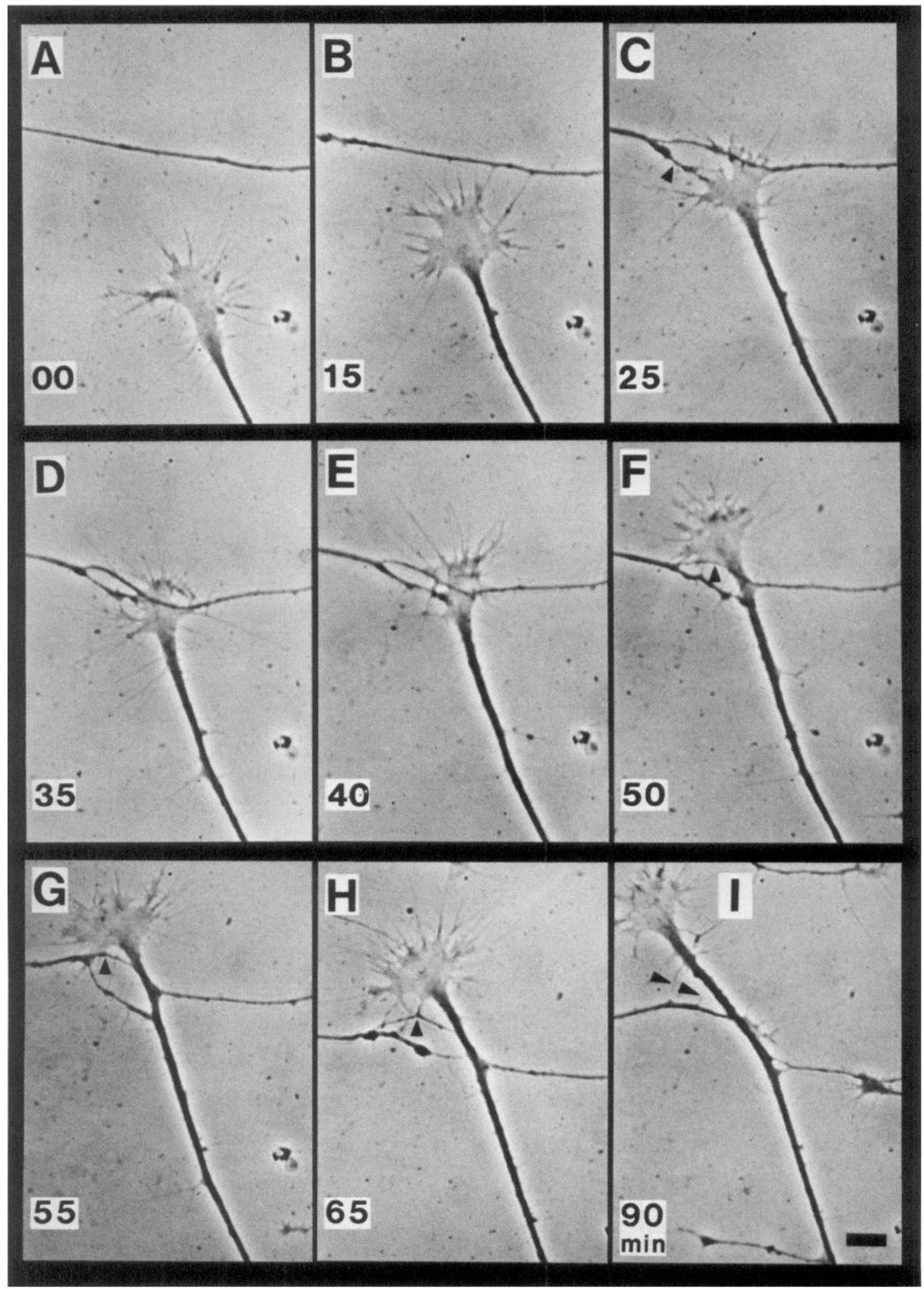

Figure 3. Sympathetic growth cone crossing a sympathetic neurite. Field shifts upwards and to the left between $G$ and $H$. Time in minutes indicated at lower left of each frame. Calibration bar, $10 \mu \mathrm{m}$. 


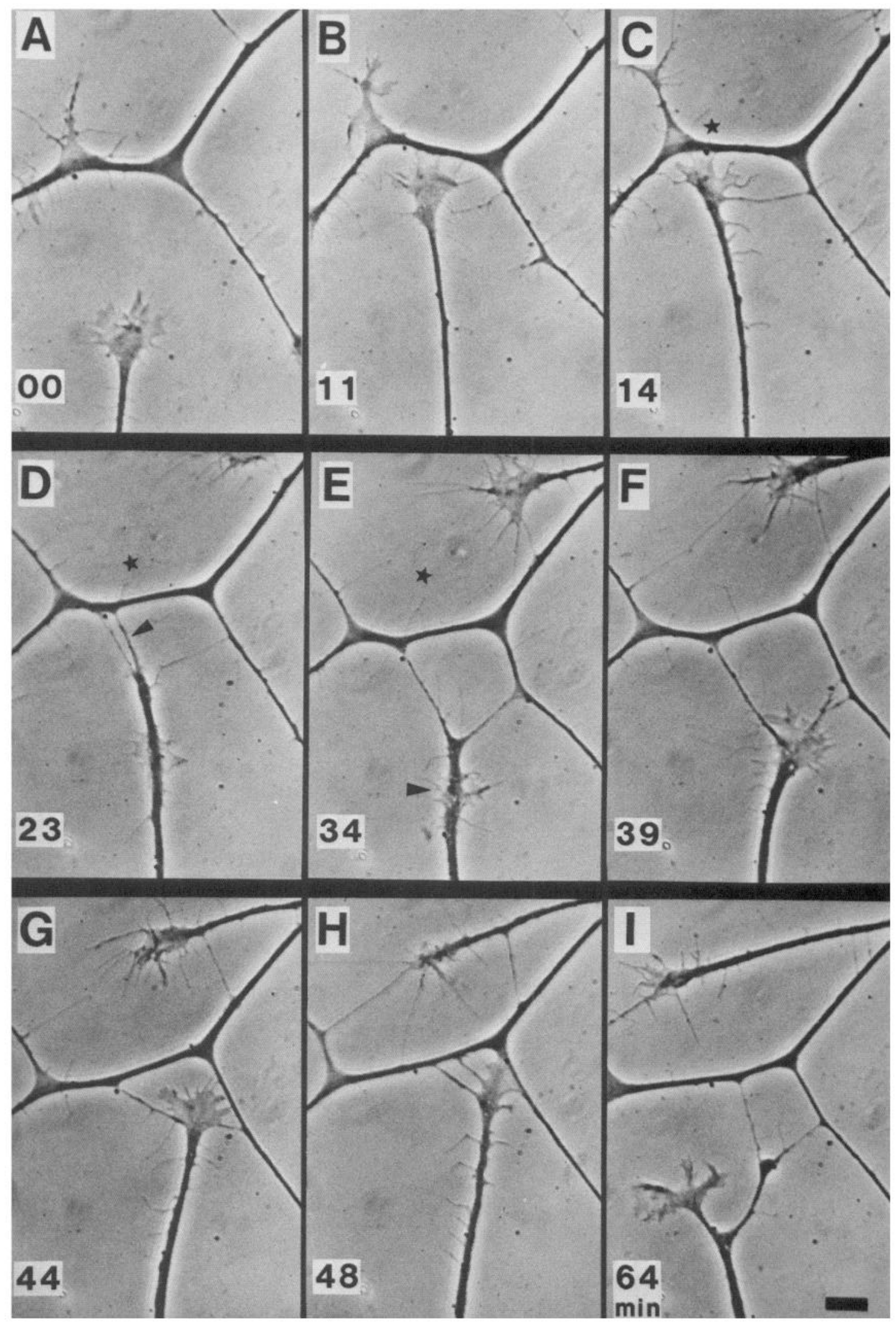

Figure 4. Retinal growth cone meeting and retracting from a sympathetic neurite. Field shifts to the left between $A$ and $B$. Time in minutes indicated at lower left of each frame. Calibration bar, $10 \mu \mathrm{m}$. 


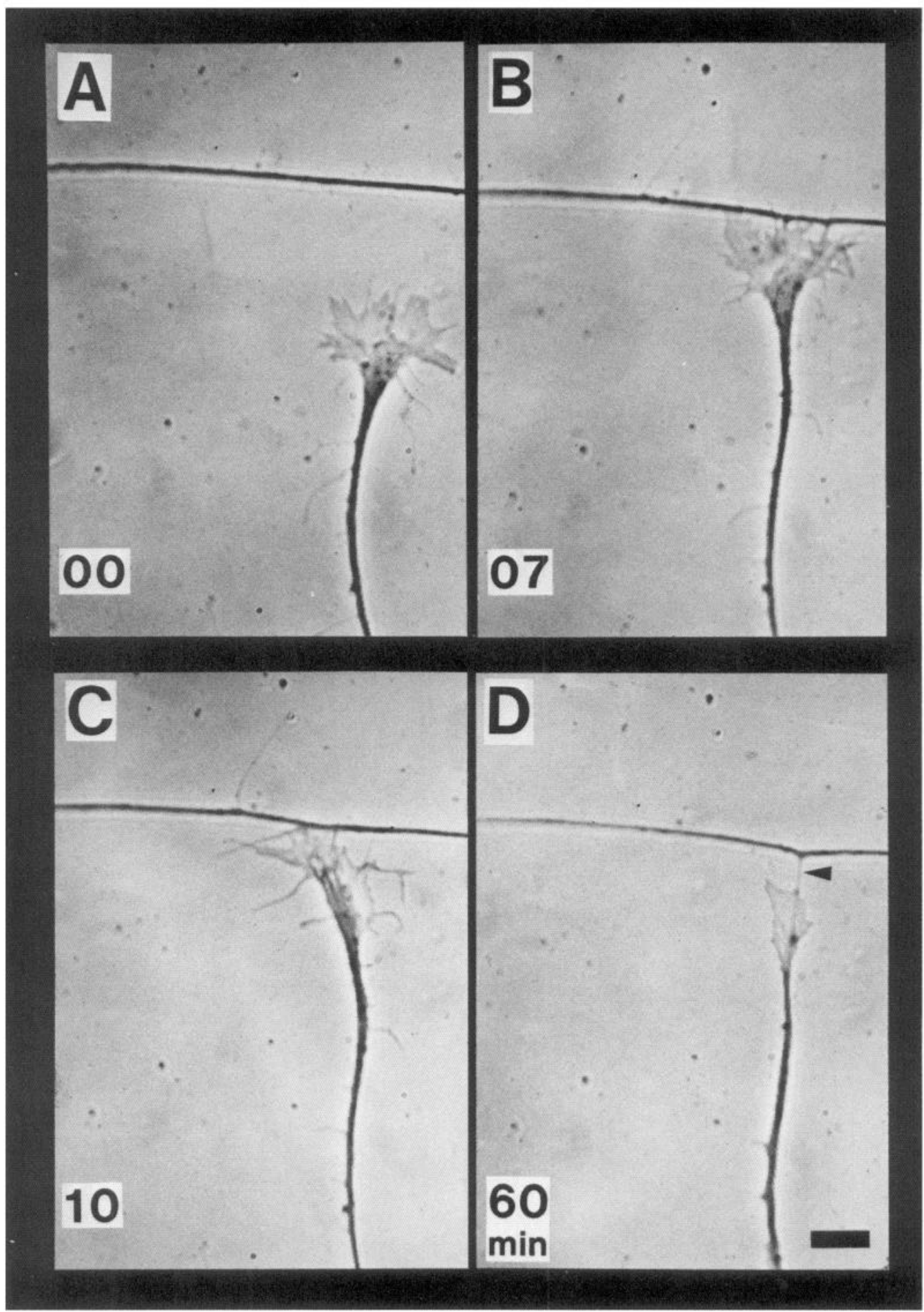

Figure 5. Retinal growth cone meeting a sympathetic neurite. In this example, retraction is incomplete, and the growth cone never recovers normal motility. Time in minutes indicated at lower left of each frame. Calibration bar, $10 \mu \mathrm{m}$.

Three minutes later, the flattened region of the growth cone has begun to shrink and the filopodia have thickened (Fig. 5C). This growth cone retracted little, if at all, and did not recover its normal flattened morphology or its ability to translocate for the remainder of the recording period (more than $1 \mathrm{hr}$ ). Instead, it remained in a collapsed, nonmotile state, conserving a thin strand of retinal tissue between itself and the sympathetic neurite (arrowhead, Fig. 5D). This pattern of behavior is analogous to that depicted in Figure 5, B3 and D3 in Kapfhammer et al. (1986).

\section{Sympathetic growth cones meeting retinal neurites}

An encounter between a sympathetic growth cone and a thin retinal neurite is shown in Figure 6 . The growth cone advances rapidly (Fig. 6A) until it has extensive filopodial and limited lamellipodial contact with the retinal neurite (Fig. $6 B$, closed 


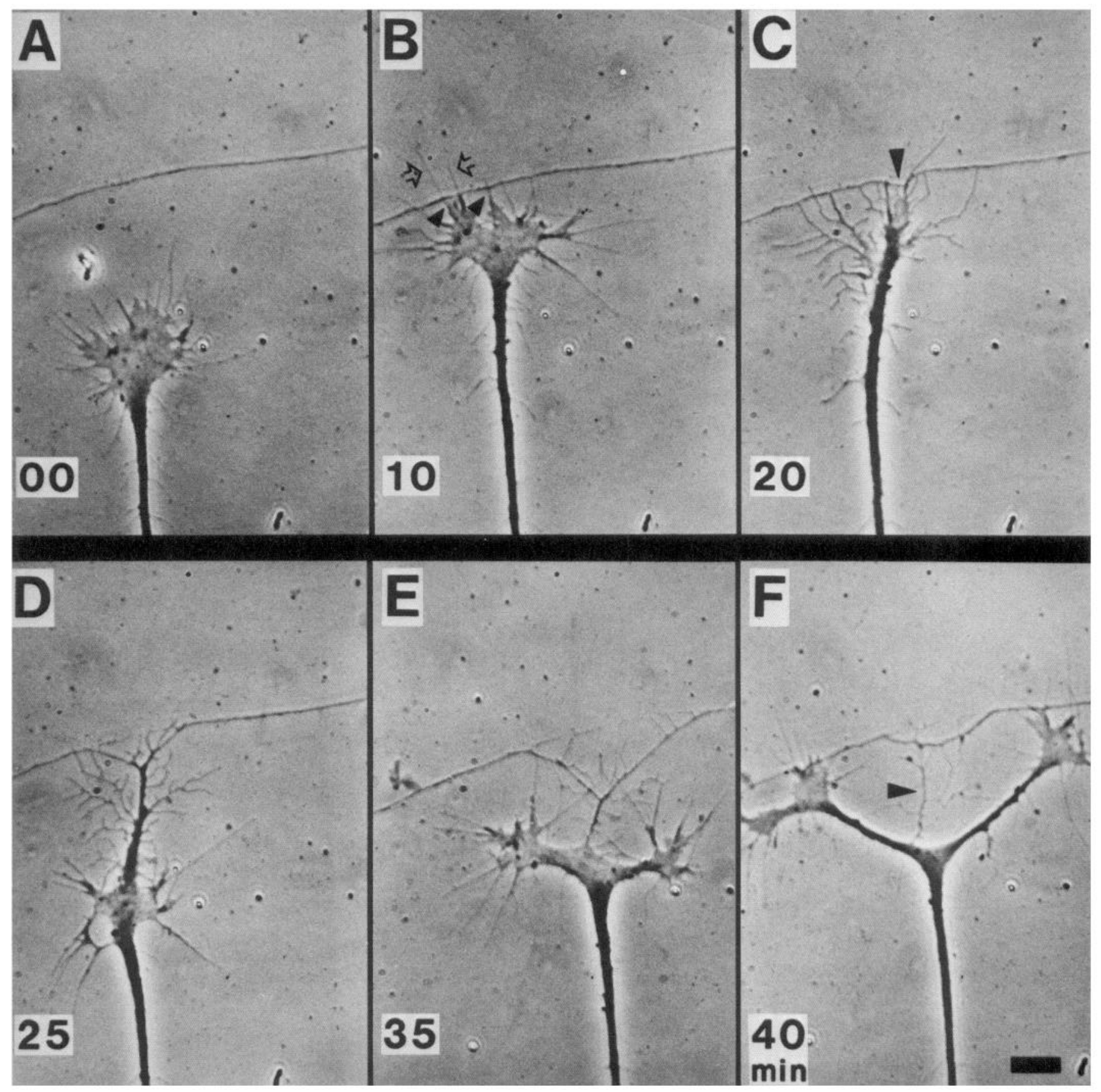

Figure 6. Sympathetic growth cone meeting and retracting from a retinal neurite. Field shifts to the left between $D$ and $E$. Time in minutes indicated at lower left of each frame. Calibration bar, $10 \mu \mathrm{m}$.

arrowheads). Some sympathetic filopodia extend beyond the retinal neurite (open arrowheads). During the next $15 \mathrm{~min}$ the sympathetic growth cone collapses in the same way that retinal growth cones collapse on contact with sympathetic neurites. The flattened region of the growth cone rapidly shrinks and a marked thickening of the filopodia occurs (Fig. 6C). The filopodia are also more branched than under normal conditions. A localized portion of the sympathetic growth cone may be actively retracting, since it is pulling the retinal neurite downwards before the sympathetic growth cone retracts as a whole (arrowhead). Within $5 \mathrm{~min}$, the tip of the sympathetic neurite is maximally re- tracted and is pulling the retinal neurite away from its original position (Fig. 6D). The sympathetic tip is resorbed by a newly forming growth cone behind it. This growth cone ultimately splits to form 2 separate growth cones (Fig. $6 E$ ), each of which readvances towards the retinal neurite (Fig. $6 F$ ). A tiny sympathetic process spans the gap between the sympathetic branch point and the original site of sympathetic-retinal contact (arrowhead).

Another example of a sympathetic growth cone meeting a retinal neurite is shown in Figure 7. Here a small sympathetic growth cone approaches (Fig. 7A) and makes filopodial contact 
Figure 7. Sympathetic growth cone meeting and retracting from a retinal neurite. In this case, retraction commenced just after filopodial contact. Time in minutes indicated at lower right of each frame. Calibration bar, $10 \mu \mathrm{m}$.

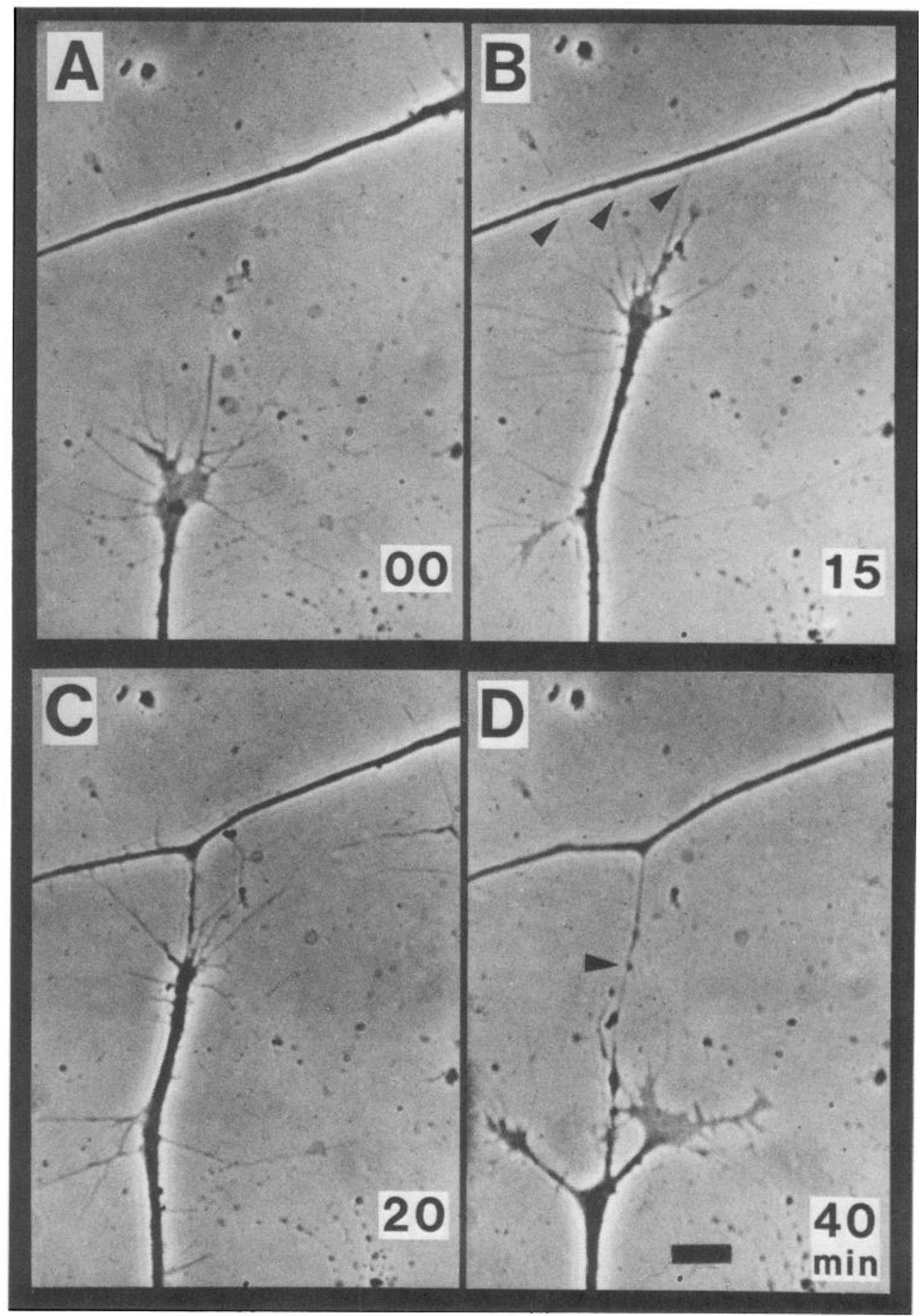

with a retinal neurite (Fig. $7 B$, arrowheads). In this particular case, filopodial contact alone precedes a collapse in growth cone structure and the retraction of the sympathetic neurite (Fig. 7, $B, C)$. Twenty minutes later, further retraction has occurred (Fig. $7 D$ ). The sympathetic neurite tip has been resorbed, leaving behind a thin sympathetic process that connects the sympathetic and retinal neurites (arrowhead). This process adheres firmly to the retinal neurite, displacing it from its original position. Two new growth cones have formed and are advancing in opposite directions.

\section{Retinal growth cones meeting sympathetic growth cones}

We have found that retinal growth cones collapse and retract upon contact with sympathetic neurites and that sympathetic growth cones collapse and retract upon contact with retinal neurites. The reciprocity of these responses is further demonstrated by the collision of a retinal growth cone with a sympathetic growth cone (Fig. 8). A retinal growth cone (top) and a sympathetic growth cone (bottom) approach each other head-on (Fig. $8 A$ ). Ten minutes later there is intensive contact between 


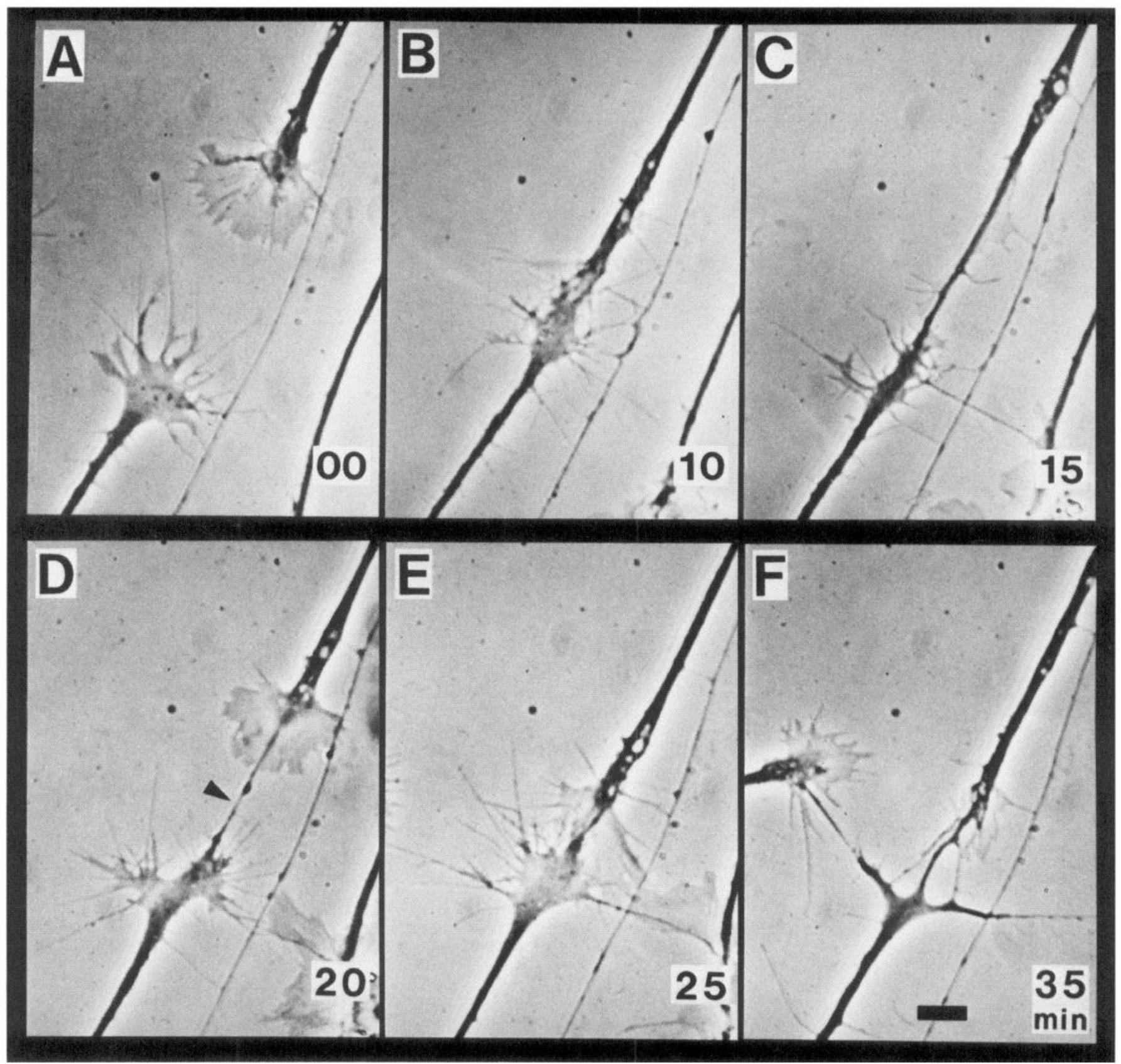

Figure 8. Retinal growth cone (top) meeting a sympathetic growth cone (bottom). Time in minutes indicated at lower right of each frame. Calibration bar, $10 \mu \mathrm{m}$.

them (Fig. 8B). Both growth cones collapse, and the retinal neurite retracts (Fig. $8 C$ ). Both neurites form new growth cones (Fig. $8 D$ ). A thin process formed during the collision connects the 2 growth cones (arrowhead in Fig. $8 D$ ). The growth cones reapproach each other and meet once again (Fig. $8 E$ ). For the second time, both growth cones collapse (Fig. $8 F$ ). These observations suggest that the labels that initiate growth cone collapse are associated with both neurites and growth cones.

\section{Establishment of exclusive retinal and sympathetic territories}

In Figure 9 is a collection of schematized sketches taken from a video recording in which a single retinal neurite, surrounded by sympathetic neurites, establishes and defends an exclusive territory. A retinal neurite (Fig. $9 A$, thicker lines and blackened growth cones) has grown into an area populated predominantly by sympathetic neurites (thinner lines and stippled growth cones). The retinal neurite has 2 branches. Branch "a" has already contacted a sympathetic neurite and is in a retracted state. Branch "b" is headed by an active growth cone and is just about to collide with both sympathetic growth cone "d" and sympathetic neurite "e" (Fig. 9A). The growth cone of retinal branch b collapses and retracts when it contacts sympathetics $d$ and e (Fig. $9 B$ ). The sympathetic growth cone $d$ branches during the same encounter (Fig. 9B). The lower branch touches retinal branch b 

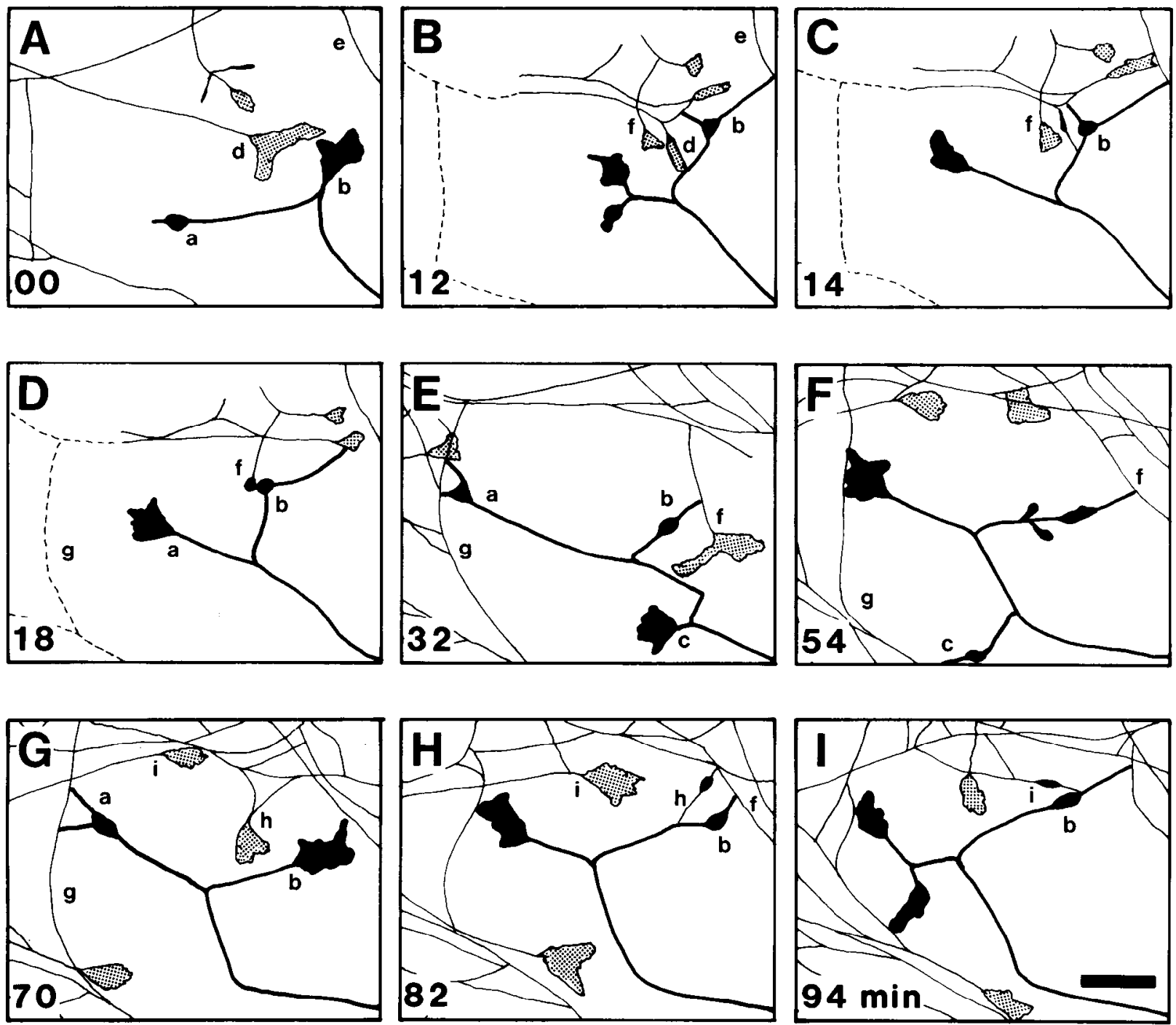

Figure 9. Establishment and defense of an exclusive territory by a retinal neurite surrounded by sympathetic neurites. Schematic representations of scenes from a videotape. Heavy lines and filled growth cones represent a retinal neurite. Thinner lines and stippled growth cones represent sympathetic neurites. Motile growth cones are represented as large and flattened; collapsed growth cones as rounded balls. a-c label branches of the retinal neurite; $d-i$ label individual sympathetic growth cones and neurites. $B-D$. The videotape was taken at a higher magnification than in the other frames. The approximate positions of some important sympathetic neurites are indicated by dashed lines in the regions out of view. Time in minutes indicated at lower left of each frame. Calibration bar, $20 \mu \mathrm{m}$.

and collapses (Fig. 9C). Sympathetic growth cone f advances towards the retinal neurite from above and collapses after contacting it (Fig. 9, $B-D$ ).

In the meantime, retinal branch a regenerates its formerly collapsed growth cone, advances, and collapses after contacting sympathetic neurite $\mathrm{g}$ (Fig. $9 E$ ). A third retinal branch, c, develops. Sympathetic neurite $f$ reforms an active growth cone, which slips by the retracting retinal branch $b$ (Fig. $9 E$ ). It then divides into 2 branches (not shown). One branch touches the retinal neurite and is retracted. The other branch pulls its trailing neurite well away from the retinal neurite (Fig. $9 F$ ). The newly formed retinal growth cone $\mathrm{c}$ retracts after contacting sympathetic neurite $\mathrm{g}$ (Fig. 9F).

By this time the retinal neurite has established a territory free of sympathetic neurites. This territory develops as sympathetic growth cones touch the retinal neurite, collapse, and retreat into sympathetic territory [e.g., $d$ (not labeled)] in Fig. $9 \mathrm{C}$ and $\mathrm{f}$ in
Fig. $9 D$ ), or as sympathetic growth cones are deflected from the retinal neurite (f in Fig. 9, $E, F$ ). The same mechanism in reverse confines the retinal neurite within its own territory as its growth cones collapse and retract upon contacting sympathetic neurites (b in Fig. 9B, a in Fig. 9E, $\mathrm{c}$ in Fig. $9 F$ ).

This territory is maintained by the same mechanisms (Fig. 9, $G-I$ ). The invading sympathetic growth cone $\mathrm{h}$ retracts after contacting retinal branch b (Fig. $9 H$ ). Another invading sympathetic growth cone, $i$, collapses and retracts after contacting the same retinal branch (Fig. 9I). A growth cone on retinal branch a collapses after contacting sympathetic $\mathrm{g}$ (Fig. $9 G$ ). A growth cone on retinal branch $b$ collapses after contacting sympathetic $\mathrm{f}$ (Fig. 9H).

\section{Discussion}

We have used cultured embryonic chick tissues to show that retinal growth cones cross retinal neurites more easily than they 
cross sympathetic neurites, while sympathetic growth cones cross sympathetic neurites more easily than they cross retinal neurites (Kapfhammer et al., 1986). This implies that retinal and sympathetic neurites have differential labels associated with them, and that retinal and sympathetic growth cones are able to distinguish between these labels. With a view toward better understanding the mechanisms underlying these specific interactions between growth cones and neurites, we have examined the morphology of retinal and sympathetic growth cones as they meet retinal or sympathetic neurites.

When retinal growth cones contact retinal neurites, or when sympathetic growth cones contact sympathetic neurites, the growth cones maintain their normal, flattened, motile morphology. In contrast, retinal growth cone morphology collapses on contact with sympathetic neurites, and sympathetic growth cone morphology collapses on contact with retinal neurites. We have never observed growth cones collapsing without first having contacted a neurite. Contact leads first to a shortening and broadening of filopodia. Then the large flattened region of the growth cone is resorbed into its own trailing neurite. As the structure of the growth cone collapses, the growth cone loses its grip on the substrate and generally retracts in the direction from which it came. Fine cytoplasmic strands originating from the collapsing growth cone continue to adhere to the incompatible neurite that initiated the collapse. Once the trailing neurite has fully retracted, a new growth cone is organized and advances.

Symmetrical growth cone branching sometimes does occur after retraction (Figs. 6, 7). This phenomenon could be related to the observation of Bray (1979) that symmetric branching becomes more probable if the tension exerted on a growth cone by its trailing neurite is reduced. Retraction, by relieving tension on the trailing neurite, could then induce a newly forming growth cone to form symmetric branches. Much more common is asymmetric branching (Figs. 4, 9), in which a new, active growth cone sprouts from the side of a neurite with an old, collapsed growth cone.

The collapse and reorganization of growth cones occur relatively quickly. The first changes in growth cone morphology occur within minutes of the initial contact between an advancing growth cone's lamellipodia and an incompatible neurite. Retractions are generally completed within 15-20 min. A second growth cone often advances within the next $10 \mathrm{~min}$, beginning the process again.

Because these results cannot be explained by the assumption that neurites are acting as passive barriers (Kapfhammer et al., 1986), it is necessary to assume that growth cones are in some way able to recognize specific neurites. Some of the mechanisms that could be responsible for this kind of recognition include diffusible signals, selective substrate modifications, differential adhesion, or contact-mediated inhibition of locomotion.

\section{Diffusible factors}

Haydon et al. (1984) have shown that a freely diffusible neurotransmitter, $5-\mathrm{HT}$, reversibly blocks the motility of a specific molluscan growth cone in culture. 5-HT causes the large flattened region of this growth cone to shrink in size, filopodial and ruffling activity to cease, and the forward motion of the growth cone to halt. These changes in growth cone morphology are similar to those we see when incompatible growth cones and neurites meet. Although we cannot definitively exclude this mechanism as an explanation for the behavior we have described, it is not likely that the release of neurotransmitters or other freely diffusible factors is involved. In every case in which we observed growth cone collapse and retraction, contact was first established between the surfaces of a growth cone and an incompatible neurite $(n=36)$. Since we never observed "action at a distance," even when growth cones approached concentrated masses of incompatible neurites, it is likely that growth cone collapse and retraction are contact-mediated.

\section{Selective substrate modification}

Chick dorsal root ganglion (DRG) neurites are able to interact intimately with mesenchymal cells from dermis, but the same neurites avoid epidermal cells (Verna, 1985). Avoidance occurs before DRG neurites touch epidermal cells, especially if polylysine is used as the culture substrate. Avoidance does not occur if the epidermal cells are dead. These results imply that epidermal cells secrete a diffusible factor that repels DRG neurites. Verna suggests that this factor modifies polylysine substrates and that DRG neurites prefer not to grow on the modified substrates.

Sympathetic and retinal neurites could identify themselves to approaching growth cones in our cultures by the secretion of neurite-specific, substrate conditioning factors. If these factors were diffusible, like those described by Verna (1985), growth cone retraction would not always appear to be contact-mediated. Even insoluble molecules extruded into the immediate vicinity of neurites are unlikely to cause the growth cone collapse observed. When neurites are pulled from one position to anotherfor example, by the activity of nearby growth cones-nothing that affects growth cone behavior appears to be left behind. Collapse occurs when a growth cone touches an incompatible neurite, not when a growth cone crawls over the former location of an incompatible neurite.

Growth cones can secrete proteases (Krystosek and Seeds, 1981). If the growth cones or neurites of particular tissues secreted different proteases, it might be possible for them to selectively modify specific components of the substrate bencath them. This, in turn, could affect the behavior of later-arriving growth cones trying to grow on the affected substrate. Our explants grow on a defined laminin substrate. For there to be any hope of obtaining the required specificity, the motility of retinal and sympathetic growth cones would have to be mediated by different parts of the laminin molecule or by different serum components bound to laminin or unblocked glass. Otherwise, there could be no specific substrate components for proteases to modify selectively. Apart from these considerations, it would be relatively difficult to explain our results by protease action. Diffusible proteases should be able to act at a distance; but, again, growth cone retraction always appears to be contactmediated. Protease action would leave immobile active patches on the substrate. However, we have no evidence thus far that active regions are left behind when a neurite is moved.

\section{Adhesion}

It has been hypothesized that growth cones prefer to grow on substrates to which they are more adherent (Letourneau, 1975; Bray, 1982). When Bray et al. (1980) originally described the tendency for retinal and sympathetic neurites to maintain separate territories in culture, they tentatively suggested that differential adhesion might be the cause. For example, if growth cones adhere to like neurites better than to unlike neurites, then growth cones might be able to grow more easily on or over like neurites than unlike neurites. This behavior would tend to gen- 
erate separate retinal and sympathetic territories.

Retinal and sympathetic growth cones have difficulty crossing unlike neurites, but they are able to adhere to them. When a growth cone collapses and retracts from an incompatible neurite, a portion of the collapsing growth cone nearly always remains attached to the neurite. This indicates that portions of the growth cone adhere to the incompatible neurite before retraction begins. These attachments remain intact even under the considerable tension associated with retraction, indicating strong adhesion. It is also unlikely that growth cones have difficulty crossing unlike neurites as a consequence of their adhering too tightly to them. One might, if this were the case, expect that the whole of a growth cone would attempt to crawl onto an incompatible neurite and then remain in place or crawl along the neurite. Growth cone collapse and retraction would not be expected. In general, any hypothesis that attempts to explain retraction by the adhesive properties of the participating tissues is unattractive, because it does not directly explain why growth cones collapse upon contact with specific neurites.

\section{Contact inhibition}

A variety of motile, non-neuronal cells prefer not to crawl over one another when they touch in culture (see Heaysman, 1978). Their net motility is hindered upon such contact. This phenomenon has been called "contact inhibition" (Abercrombie and Heaysman, 1954). Depending on the cell types involved, either of 2 mcchanisms can lead to contact inhibition. For cxamplc, the net motion of transformed rat fibroblasts is inhibited upon contact with one another (Vesely and Weiss, 1973). The membranes of these cells continue to express their normal motile morphology and activity, but the cells are unable to crawl over one another, presumably as a result of their inability to adhere to one another. This phenomenon was named "contact inhibition type II" by Vesely and Weiss (1973). It is analogous to an explanation based on differential adhesion and, for the reasons already discussed, provides an unsatisfactory explanation for our observations.

Other cells, for example, chick heart fibroblasts (Abercrombie and Heaysman, 1954; Abercrombie and Ambrose, 1958), display another form of contact inhibition (type I, Vesely and Weiss, 1973). These cells first adhere to one another on contact (Abercrombie, 1970; Heaysman, 1978). Their locomotory behavior soon becomes paralyzed in the region of contact, and they withdraw from one another. Strands of stretched cytoplasm usually span the gaps between retreating cells. The cells recover once they are no longer in contact, and resume normal locomotion until they collide again.

These events exactly parallel those we observe when retinal growth cones meet sympathetic neurites or when sympathetic growth cones meet retinal neurites. Interestingly, we have observed this kind of contact inhibition only when growth cones meet neurites from a different neural tissue, while the same behavior is often observed between non-neuronal cells from the same tissue. This pattern is consistent with the observation that contact inhibition does not occur between ciliary growth cones and ciliary neurites, or between DRG growth cones and DRG neurites (Wessells et al., 1980).

It has been hypothesized that contact inhibition occurs when a signal is passed between colliding cells. This signal could then trigger each cell's avoidance response (Abercrombie, 1970). The signal could be mediated by a diffusible substance only if its concentration gradient were extremely steep. If one accepts the hypothesis that avoidance is contact-mediated, then the signal could be a cell-surface-cell-surface recognition event. Alternatively, the signal could involve the exchange of intracellular components through gap junctions or cytoplasmic bridges joining the cells' interiors. The interiors of actively locomoting growth cones have been shown in the grasshopper embryo, using a fluorescent tracer, to exchange contents with the interiors of specific subsets of cells (Bate and Grunewald, 1981; Raper and Goodman, 1982; Taghert et al., 1982).

Our results suggest that the motility of particular growth cones is shut down if they contact specific neurites. The role this phenomenon might play in vivo is potentially interesting. Given a choice, growth cones probably prefer to fasciculate on neurites that do not cause them to collapse. Selective fasciculation would be the result. More generally, repulsive interactions could be useful in canalizing growth cones into their appropriate routes. Finally, targets might capture innervating neurites by signaling their growth cones to shut down on contact.

\section{References}

Abercrombie, M. (1970) Contact inhibition in tissue culture. In Vitro 6: $128-142$.

Abercrombie, M., and E. J. Ambrose (1958) Interference microscope studies of cell contacts in tissue culture. Exp. Cell Res. 15: 332-345.

Abercrombie, M., and J. E. M. Heaysman (1954) Observations on the behavior of cells in tissue culture: II. Monolayering of fibroblasts. Exp. Cell Res. 6: 293-306.

Bate, C. M., and E. B. Grunewald (1981) Embryogenesis of an insect nervous system. II. A second class of neuron precursor cells and the origin of the intersegmental connectives. J. Embryol. Exp. Morphol. 61: 317-330.

Bray, D. (1979) Mechanical tension produced by nerve cells in tissue culture. J. Cell Sci. 37: 391-410.

Bray, D. (1982) Filopodial contraction and growth cone guidance. In Cell Behaviour, R. Bellairs, A. Curtis, and G. Dunn, eds., pp. 299317, Cambridge U. P., Cambridge, UK.

Bray, D., P. Wood, and R. P. Bunge (1980) Selective fasciculation of nerve fibers in culture. Exp. Cell Res. 130: 241-250.

Haydon, P. G., D. B. McCobb, and S. B. Kater (1984) Serotonin selectively inhibits growth cone motility and synaptogenesis of specific identified neurons. Science 226: 561-564.

Heaysman, J. E. M. (1978) Contact inhibition of locomotion: A reappraisal. Int. Rev. Cytol. 55: 49-66.

Kapfhammer, J. P., B. E. Grunewald, and J. A. Raper (1986) The selective inhibition of growth cone extension by specific neurites in culture. J. Neurosci. 6: 2527-2534.

Krystosek, A., and N. W. Seeds (1981) Plasminogen activator release at the neuronal growth cone. Science 213: 1532-1534.

Letourneau, P. C. (1975) Cell-to-substratum adhesion and guidance of axonal elongation. Dev. Biol. 44: 92-101.

Nishi, R., and D. K. Berg (1977) Dissociated ciliary ganglion neurons in vitro: Survival and synapse formation. Proc. Natl. Acad. Sci. USA 74: $5171-5175$.

Raper, J. A., and C. S. Goodman (1982) Transient dye coupling between developing neurons reveals patterns of intercellular communication during embryogenesis. In Cellular Communication During Ocular Development, J. B. Sheffield and S. R. Hilfer, eds, pp. 85-96, Springer, New York.

Taghert, P. H., M. J. Bastiani, R. K. Ho, and C. S. Goodman (1982) Guidance of pioneer growth cones: Filopodial contacts and coupling revealed with an antibody to Lucifer Yellow. Dev. Biol. 94: 391-399.

Verna, J.-M. (1985) In vitro analysis of interactions between sensory neurons and skin: Evidence for selective innervation of dermis and epidermis. J. Embryol. Exp. Morphol. 86: 53-70.

Vesely, P., and R. A. Weiss (1973) Cell locomotion and contact inhibition of normal and neoplastic rat cells. Int. J. Cancer 11:64-76.

Wessells, N. K., P. C. Letourneau, R. P. Nuttall, M. Luduena-Anderson, and J. M. Geiduschek (1980) Responses to cell contacts between growth cones, neurites and ganglionic non-neuronal cells. J. Neurocytol. 9: 647-664. 